Technical note

\title{
Dosimetric and radiation cancer risk evaluation of high resolution thorax CT during COVID-19 outbreak
}

\author{
C. Ghetti ${ }^{\text {a }}$, O. Ortenzia ${ }^{\text {a, }}$, M. Maddalo ${ }^{\text {a }}$, L. Altabella ${ }^{\text {a }}$, N. Sverzellati ${ }^{\text {b }}$ \\ ${ }^{a}$ Department of Medical Physics, University Hospital of Parma, Italy \\ ${ }^{\mathrm{b}}$ Department of Medicine and Surgery, Division of Radiology, University of Parma, Italy
}

\section{A R T I C L E I N F O}

\section{Keywords:}

CT dosimetry

Thorax imaging

COVID-19

Dose tracking

Lifetime attributable risk

\begin{abstract}
A B S T R A C T
Purpose: The aim of this work was to evaluate the dosimetric impact of high-resolution thorax CT during COVID19 outbreak in the University Hospital of Parma. In two months we have performed a huge number of thorax CT scans collecting effective and equivalent organ doses and evaluating also the lifetime attributable risk (LAR) of lung and other major cancers.

Materials and Method: From February 24th to April 28th, 3224 high-resolution thorax CT were acquired. For all patients we have examined the volumetric computed tomography dose index (CTDIvol), the dose length product (DLP), the size-specific dose estimate (SSDE) and effective dose ( $\left.\mathrm{E}_{103}\right)$ using a dose tracking software (Radimetrics Bayer HealthCare). From the equivalent dose to organs for each patient, LAR for lung and major cancers were estimated following the method proposed in BEIR VII which considers age and sex differences. Results: Study population included 3224 patients, 1843 male and 1381 female, with an average age of 67 years. The average CTDIvol, SSDE and DLP, and $\mathrm{E}_{103}$ were $6.8 \mathrm{mGy}, 8.7 \mathrm{mGy}, 239 \mathrm{mGy} \cdot \mathrm{cm}$ and $4.4 \mathrm{mSv}$ respectively. The average LAR of all solid cancers was 2.1 cases per 10,000 patients, while the average LAR of leukemia was 0.2 cases per 10,000 patients. For both male and female the organ with a major cancer risk was lung. Conclusions: Despite the impressive increment in thoracic CT examinations due to COVID-19 outbreak, the high resolution low dose protocol used in our hospital guaranteed low doses and very low risk estimation in terms of LAR.
\end{abstract}

\section{Introduction}

The new emerging viral pneumonia named COVID-19 (Coronavirus Disease 2019) related to the novel coronavirus SARS-CoV-2 (Severe Acute Respiratory Syndrome Coronavirus 2) is affecting several countries in particular Northern Italy with a dramatic outbreak from the end of February 2020 [1,2].

The reference standard for the diagnosis of SARS-CoV-2 infection is the collection of respiratory tract specimens, analysed using nextgeneration sequencing or real-time reverse transcription polymerase chain reaction (RT-PCR) methods [3], with a sensitivity ranged between $60 \%$ and $71 \%$ [4-7]. Several works reported that the chest CT to detect COVID-19 pneumonia guaranteed superior sensitivity (around 97\%) with respect to standard reference $[5,6,7,8]$. This has initially suggested that CT imaging may be helpful in early detection of COVID-19 suspected interstitial pneumonia patients. Latest recommendations proved that there is no general consensus in the use of CT for COVID-19 diagnosis because in the first stage of the disease it could be negative [9]. More precisely, European Radiology Society (ESR) and European Society of Thoracic Imaging (ESTI) suggested using CT imaging to stratify patients selected from first-line clinical trials [9]. This because CT imaging, with respect to chest radiography, is more sensitive to ground glass opacity, which is the principle imaging feature of COVID-19 pneumonia. Chest radiography is instead recommended in patient follow up [9].

In our Institution more than three thousand high-resolution thorax CT were performed from the beginning of the outbreak to help diagnosis and staging of COVID-19 pneumonia [10]. Such a big number required also for a couple of weeks an installation of a further mobile CT in order to meet the increasing demand for such methodology.

This impressive and sudden increase of this diagnostic investigation could lead to dosimetric consequences in patients and low dose protocols for chest CT in patients suspected with COVID-19 pneumonia are recommended [11]. Indeed, there is concern that exposure to the

\footnotetext{
* Corresponding author.

E-mail address: oortenzia@ao.pr.it (O. Ortenzia).
} 
ionising radiation of CT might increase the risk of developing solid cancers and leukaemia [12].

The aim of this study was the evaluation of the dosimetric impact of high resolution thorax CT during COVID-19 outbreak in our Institution. For this reason, as first analysis, mean organ doses and effective doses were evaluated. Then, we investigated the lifetime attributable risk (LAR) of cancer incidence associated with these exposures according to National Research Council Biologic Effects of Ionizing Radiation (BEIR) VII report [13].

\section{Materials and methods}

\subsection{Study population}

Between February 24th and April 28th 2020, 3224 high-resolution (HR) thorax CT scans were performed at the University Hospital of Parma for suspected SARS-CoV-2 infection.

Due to the evolution and the emergence of the disease, the care pathways have been modified during the time of pandemic and, for this reason, the patients have been directed to different CT systems. Separate diagnostic pathways were expressly developed for those patients due to confinement requirements and for this reason, data collection was extremely simplified and no exclusion criteria were needed.

Moreover, in support during most critical weeks, a mobile CT was also rented allowing to increase the number of exams daily.

\subsection{CT equipment and techniques}

The images were collected on two Somatom Definition Edge Scanner (Siemens Healthcare, Germany) equipped with Stellar detector and Admire iterative reconstruction, both installed in 2019 and on Somatom Emotion 16 equipped with Ultra Fast Ceramic (UFC ${ }^{\mathrm{TM}}$ ) detector installed on wheels (2007).

Given the sudden outbreak of the COVID-19 epidemic a pre-existing Thorax HR protocol commonly used to investigate patients with suspected idiopathic interstitial pneumonia has been performed for suspected COVID-19-patient lungs. During the same period of the previous year (February 24th and April 28th 2019) only 405 CT Thorax HR examinations were acquired instead of 3224 exams.

The clinical thorax protocol was performed on Edge scanners using $120 \mathrm{kV}, 1 \mathrm{~mm}$ slice thickness, 1.2 pitch and $128 \times 0.6 \mathrm{~mm}$ collimation. Similar protocol was implemented on Emotion 16 with $110 \mathrm{kV}, 1 \mathrm{~mm}$ slice thickness, 1.2 pitch and $16 \times 0.6 \mathrm{~mm}$ collimation.

On all CT scanners, automatic exposure control named CARE Dose 4D, was turned on using effective $110 \mathrm{mAs}$.

\subsection{Data source}

Examination data were collected using a dose tracking software Radimetrics version 2.9.2b (Bayer HealthCare, Whippany, NY). Radimetrics was installed on a virtual server (OS CentOS release 6.10), on the existing RIS-PACS (Esaote) infrastructure with two vCPU, 16 GB Ram). Moreover, Radimetrics has been customized for data anonymization.

Data of CT examinations performed have been extracted from the DICOM header and CT Radiation Dose Structured Report (RDSR) files produced by the scanners and stored in PACS. The information collected includes patient information (age, gender), examination data (protocol name, number of acquisitions, name of the device), acquisition data (scan region, series name and technical parameters of each scan) and exposure data (volumetric computed tomography dose index CTDIvol, dose length product DLP). The size-specific dose estimates for body examinations SSDE, equivalent dose to organs and effective dose $\mathrm{E}_{103}$ have been extracted from Radimetrics.

\subsection{Dosimetric data}

The CTDIvol values provided by every CT scanners were verified by direct measurements in 16 and $32 \mathrm{~cm}$ diameter dedicated polymethylmethacrylate (PMMA) phantoms with a calibrated dose meter (RaySafe $^{\mathrm{TM}}$ X2 CT Sensor). A good agreement, within $10 \%$, between recorded and measured values of CTDIvol was obtained for all CT scanners and scan thickness.

The mean organ dose calculation of the CT scans was performed using the Radimetrics software by pre-run Montecarlo simulations utilizing 20 types of Cristy phantoms.

Using the scanogram information, a suitable phantom is matched automatically to the patient and the calculation is adapted to the CT acquisition parameter. The equivalent doses to the organs were then used to calculate the effective dose $\mathrm{E}_{103}$ according to the tissue weighting factors reported in ICRP 103 [14].

The equivalent doses to the following organs were reported: stomach, colon, liver, lung, bladder, thyroid, red marrow and reminder organs. Uterus and ovary were also included for female population and prostate for males.

\subsection{Estimation of cancer risk}

The lifetime attributable risks (LARs) of cancer induction were estimated from organ equivalent doses using the Biological Effects of Ionizing Radiations (BEIR) VII report. The LAR represents the incidence of solid cancers and of leukemia per 100,000 subjects exposed to 100 mSv. The BEIR VII model was chosen because it is a conservative approach (it probably overestimates risk of cancer induction at the low doses that are typical of x-ray diagnostics). The model assumes that cancer risk follows a "linear-no-threshold" (LNT) behavior also at low doses $(<100 \mathrm{mSv})$. It also considers a dose and dose-rate reduction factor (DDREF) of 1.5 for solid cancers.

Moreover, BEIR VII provided risk estimates for $100 \mathrm{mSv}$ that could be scaled linearly on the basis of the actual equivalent doses to organs received by the patient. Therefore, the relation between LAR and dose could be written as:

$\mathrm{LAR}=\operatorname{LAR} 100 \quad \frac{H}{100} / 10$

where $\mathrm{H}$ is the equivalent dose to the organ and LAR100 is the BEIR VII risk estimate. LAR values have been divided by a factor 10 , they mean the cancer incidence per 10,000 subjects.

\subsubsection{LAR estimates for the whole population}

Table 12-5A of the BEIR VII report provides organ-specific risk estimates (for bladder, breast, colon, liver, lung, ovaries, prostate, stomach, thyroid and uterus) and their uncertainties. The uncertainty of risk estimates takes into account the uncertainties related to sampling variability, to transport, and to DDREF. It does not include the modifying effects of age at exposure. Details of the confidence interval computation of risk estimates are reported in the Annex 12C of BEIR VII report. Data from table 12-5A were thus used to compute LAR for the whole population, discriminating respect to gender only. Mean values of equivalent doses for male and female subpopulations were employed. LAR uncertainty was obtained combining the equivalent dose uncertainty and the risk estimates uncertainty. Detail of LAR uncertainty calculation are described in statistical analysis subsection.

\subsubsection{Age specific LAR estimates}

In order to take into account the age at exposure in LAR computation, we have considered table 12D-1 of BEIR VII. This table provides age- and sex-specific risk estimates for several organs (bladder, breast, colon, liver, lung, ovaries, prostate, red bone marrow, stomach, thyroid and uterus).

Age-specific risk estimates were selected according to the age at 
exposure of each patient (without interpolation within categories) considering discrete (non-overlapping) age categories according to table 12D-1 of the BEIR VII report. For LAR reporting several patient subcohorts were defined based on gender and age categories. Age- and sex- specific LAR was finally averaged over the whole population to compute a global LAR estimate for solid cancer and for leukemia.

Risk estimates for individual organs tabulated in table 12D-1 of BEIR VII report lack of confidence intervals that allow a proper estimation of LAR uncertainty. Thus, 95\% confidence intervals were computed taking into account the contribution of equivalent dose uncertainty only.

\subsection{Statistical analysis}

For each dosimetric parameter (CTDIvol_Body, SSDE, DLP and E $\mathrm{E}_{103}$ ) we calculated the mean, the median value, the standard deviation and the 75th percentile. The latter was considered in order to compare our data with published values that are often reported as DRL [15]. The mean value $(\bar{H})$ and standard deviation $\left(\sigma_{H}\right)$ of equivalent doses to organs have also been determined for both gender subpopulations. The LAR standard errors were computed using the formula:

$\sigma_{\overline{L A R}}=\overline{L A R} \hat{\mathrm{A}} \cdot \sqrt{\frac{\sigma_{\bar{H}^{2}}}{\bar{H}^{2}}+\frac{\sigma_{\overline{L A R 100}^{2}}}{\overline{L A R} 100^{2}}}$,

where $\overline{L A R}$ is the mean value of LAR of the subpopulation (male or female), $s_{\bar{D}}$ is the equivalent dose standard error (i.e. the standard deviation of the mean), $\sigma_{\overline{L A R 100}}$ and $\overline{L A R 100}$ are respectively the standard error and the mean value of risk estimates tabulated in table $12-5 \mathrm{~A}$ of BEIR VII report.

$\sigma_{\bar{H}}$ was computed as follow:

$\sigma_{\bar{H}}=\frac{\sigma H}{\sqrt{n}}$

where $n$ is the subpopulation sample size.

$s_{\overline{L A R} 100}$ was determined from tabulated confidence intervals using the formula:

$s_{\overline{L A R 100}}=\frac{C I_{L A R 100}{ }^{+}-C_{L A R 100}{ }^{-}}{3.92}$

where $\mathrm{CI}_{L A R 100}{ }^{+}$and $\mathrm{II}_{\mathrm{LAR} 100}{ }^{-}$are the confidence intervals of $L A R 100$ tabulated in table 12-5A of BEIR VII report.

The $95 \%$ confidence intervals of $\overline{L A R}$ were computed multiplying the LAR standard error by a factor of 1.96 .

\section{Results}

\subsection{Study population}

Of the 3224 examined patients during a COVID-19 outbreak, 1843 (57.2\%) were men and $1381(42.8 \%)$ were women. The mean age was $67 \pm 17$ years (age range 17-105 years) and the mean weight of our

Table 1

Number of patients which belonged to each sub-cohort according to TABLE 12D1 of the BEIR VII report. In our cohort of patients nobody belongs to lower age categories (below 17.5 age).

\begin{tabular}{llll}
\hline age category $(\mathrm{y})$ & female & male & both genders \\
\hline$<17.5$ & 0 & 0 & 0 \\
$17.5-25$ & 13 & 24 & 37 \\
$25-35$ & 59 & 52 & 111 \\
$35-45$ & 105 & 101 & 206 \\
$45-55$ & 161 & 243 & 404 \\
$55-65$ & 215 & 346 & 561 \\
$65-75$ & 227 & 421 & 648 \\
$>75$ & 601 & 656 & 1257 \\
total population & $1381(42.8 \%)$ & $1843(57.2 \%)$ & $3224(100 \%)$ \\
\hline
\end{tabular}

population was $92.8 \pm 8.4 \mathrm{~kg}$. Table 1 shows demographic data for the total population and for each patient age-related sub-cohort.

\subsection{Dosimetric Data Results}

In Fig. 1 number of CT scans for suspected COVID-19 performed on a daily basis in our Hospital are shown. This number has increased during the first two weeks of March reaching a peak on the 19th of March and then decreasing until the end of April.

In Table 2 the results of statistical analysis of CT scan dosimetric parameters in terms of the mean value (MV), the median, the standard deviation $(\sigma)$ and 75th percentile (75th) are reported for total population, for females and males.

In Fig. 2 we illustrate the CTDIvol_Body and the SSDE frequency distributions and in Fig. 3 we present the frequency distributions of $E_{103}$.

For each organ, such as stomach, colon, liver, lung, bladder, thyroid, red marrow, breast, uterus and ovary for the female population and the prostate for the male population, the MV of equivalent dose to organ $(\mathrm{H})$ and the standard deviation $\left(\sigma_{\mathrm{H}}\right)$ were reported in Table 3 .

\subsection{Estimated cancer risk}

Risk estimates reported in the BEIR VII report were scaled linearly using CT exposure data which have been collected in the University Hospital of Parma during COVID-19 outbreak. Organ specific LAR for male and female subpopulation were determined using equation (1), i.e. by the product between mean equivalent doses and risk estimates of table 12-5A. Fig. 4 shows the LAR estimates for females and for males for each cancer site (Fig. 4.a). For male we observed a major risk for the lung cancer, while for female, both lung and breast cancer risks were significant. From the data of table 12D-1 we computed the mean LAR for the whole population, matching the age specific risk estimate to the corresponding age category. The mean LAR of all solid cancers was 2.1 cases per 10,000 patients, while the average LAR of leukemia was 0.2 cases per 10,000 patients (both genders, all age categories). In Fig. 4.b LAR estimates of lung cancer are reported for all age categories in order to evaluate a different behaviour with the age of exposure. LAR estimates for all other cancer types for different age categories are reported in Fig. 4.c. Confidence intervals in Fig. 4.b and 4.c are clearly underestimated. In fact, they do not take into account the uncertainty of risk estimates, which is missing in table $12 \mathrm{D}-1$.

\section{Discussion}

In this work we provide a summary of radiation dose data for chest

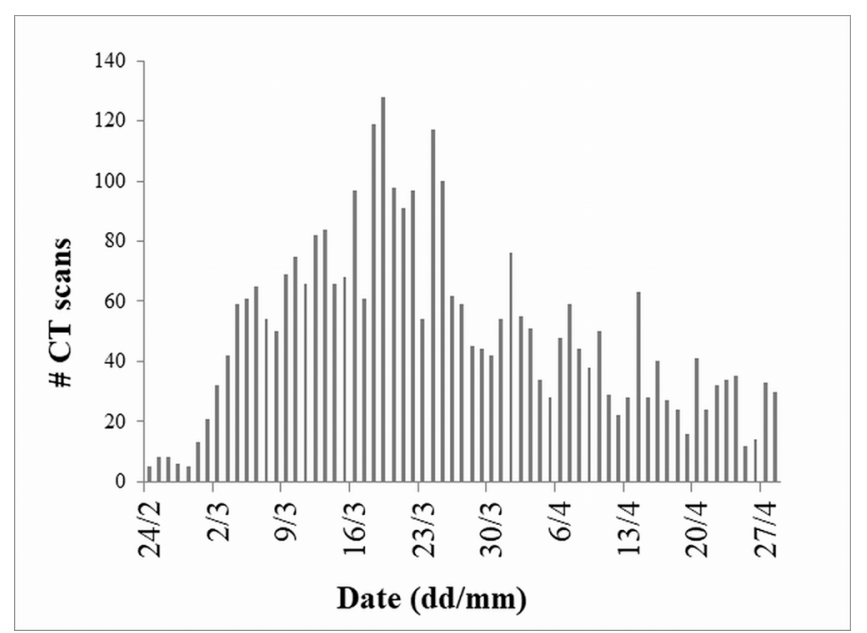

Fig. 1. The number of CT scans for suspected COVID-19 performed daily at the University Hospital of Parma from February 24th to April $28^{\text {th }}$. 
Table 2

The mean value (MV), the median, the standard deviation $(\sigma)$ and the 75 th percentile (75th) for CTDIvol_Body, SSDE, DLP_Body and $\mathrm{E}_{103}$ reported for total population, for females and males.

\begin{tabular}{lllll}
\hline Total & MV & $\sigma$ & Median & 75th \\
\hline CTDIvol_Body (mGy) & 6.8 & 2.7 & 6.2 & 8.0 \\
SSDE (mGy) & 8.7 & 3.0 & 7.8 & 9.8 \\
DLP_Body (mGy·cm) & 239 & 94 & 219 & 281 \\
E $_{103}(\mathrm{mSv})$ & 4.4 & 1.6 & 3.9 & 5.0 \\
Females & MV & $\sigma$ & Median & 75 th \\
CTDIvol_Body (mGy) & 6.5 & 3.0 & 5.8 & 8.9 \\
SSDE (mGy) & 8.8 & 3.6 & 7.7 & 10.5 \\
DLP_Body (mGy·cm) & 218 & 98 & 195 & 266 \\
E 103 (mSv) & 4.9 & 1.9 & 4.5 & 5.9 \\
Males & & & & 75 th \\
CTDIvol_Body (mGy) & 7.0 & 2.4 & 6.4 & 8.0 \\
SSDE (mGy) & 8.6 & 2.5 & 7.9 & 9.5 \\
DLP_Body (mGy·cm) & 255 & 88 & 235 & 290 \\
E $_{103}$ (mSv) & 4.0 & 1.1 & 3.7 & 4.4 \\
\hline
\end{tabular}

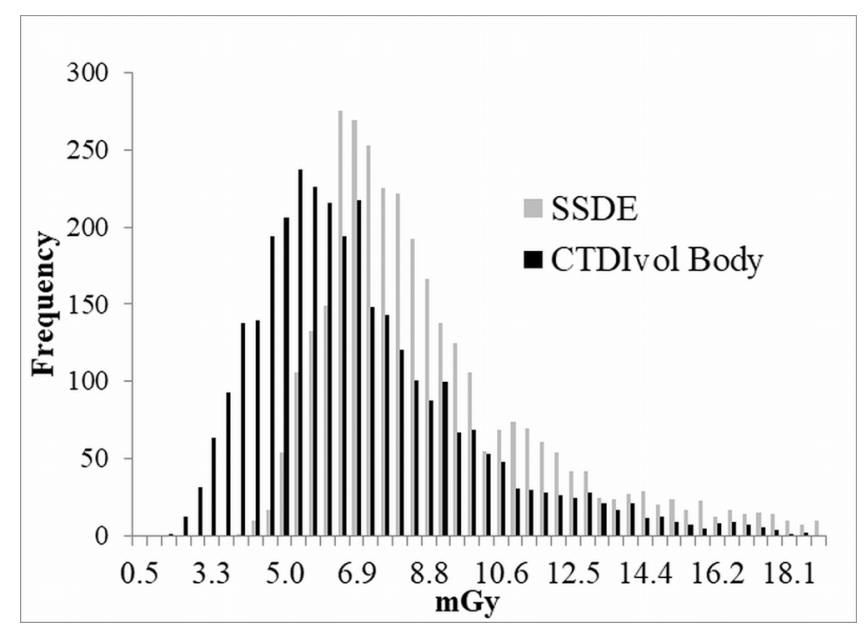

Fig. 2. SSDE and CTDIvol_Body frequency distributions.

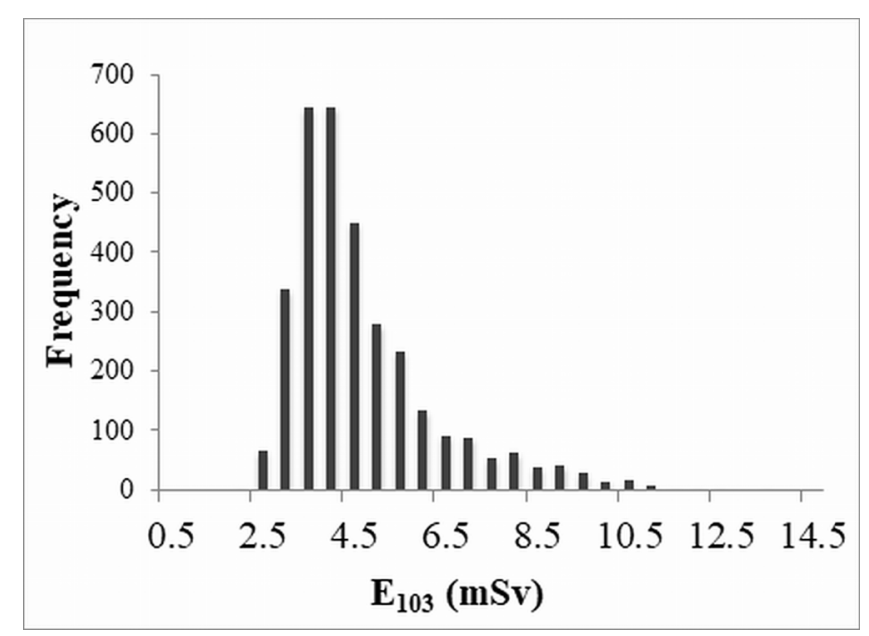

Fig. 3. E103 frequency distributions.

CT dose metrics for suspected COVID-19 pneumonia acquired during Coronavirus disease outbreak in our Institution.

Ratio between men and women and age range of our population reflects the characteristics of patients positive for COVID-19 pneumonia. Indeed, it seems that COVID-19 mostly affects men with respect to
Table 3

Equivalent dose $\mathrm{H}$ and standard deviation $\sigma_{\mathrm{H}}$ to organs for females and males.

\begin{tabular}{lll}
\hline Organ & Females & Males \\
& $\mathrm{H} \pm \sigma_{\mathrm{H}}(\mathrm{mSv})$ & $\mathrm{H} \pm \sigma_{\mathrm{H}}(\mathrm{mSv})$ \\
\hline Stomach & $6.7 \pm 2.5$ & $7.7 \pm 2.1$ \\
Colon & $0.7 \pm 0.4$ & $1.0 \pm 0.5$ \\
Liver & $7.4 \pm 2.8$ & $8.0 \pm 2.2$ \\
Lung & $9.6 \pm 3.8$ & $9.4 \pm 2.6$ \\
Bladder & $0.1 \pm 0.1$ & $0.1 \pm 0.2$ \\
Thyroid & $1.8 \pm 1.3$ & $0.9 \pm 1.0$ \\
Red marrow & $3.1 \pm 1.2$ & $3.2 \pm 1.0$ \\
Breast & $9.7 \pm 4.2$ & - \\
Uterus & $0.2 \pm 0.2$ & - \\
Ovary & $0.2 \pm 0.2$ & - \\
Prostate & - & $0.1 \pm 0.1$ \\
\hline
\end{tabular}

women with a high average age [1].

As reported in the advice from ESR [9], CT is indicated the most appropriate tool for identifying ground glass opacity of COVID-19 pneumonia. Due to the considerable increase of CT examinations for suspected patients COVID-19, we have considered interesting to evaluate the our dosimetric results in terms of DRLs, while being aware that the DRLs are estimated at a standard patient that does not match with our population composed by more overweight COVID-19 patients.

Despite the differences in the sample examined, we have verified that our medial values of the dosimetric indices are lower than national and international DRLs.

Dosimetric results in terms of CTDIvol, SSDE, DLP and $\mathrm{E}_{103}$ are comparable with patient suspected idiopathic interstitial pneumonia and are in line with previous literature of doses reported for chest CT [16]. In particular, $>50 \%$ of cases present an effective dose below 4.5 $\mathrm{mSv}$.

More precisely, our results expressed as the median value for all dosimetric indices are lower compared to those reported, in particular in [16] that summarized the CTDIvol, SSDE and DLP for International and Italian DRLs for chest CT exams [16,17,18,19,20,21]. The most recent Italian DRLs for chest exams [22] reported CTDIvol $=15 \mathrm{mGy}$ and DLP $=569 \mathrm{mGy} \cdot \mathrm{cm}$ which are largely higher than our median values (CTDIvol $=6.2 \mathrm{mGy}$ and DLP $=219 \mathrm{mGy} \cdot \mathrm{cm}$ ).

The use of an advanced software for dosimetric calculations as Radimetrics is a strength point of this work. Indeed, this software can evaluate the mean organ doses, and thus effective doses, for different groups of patients (both male and female) according to their body size.

The high dose values obtained for a small amount of patients (please refers to Fig. 3) that reach an effective dose of about $13.5 \mathrm{mSv}$ are strongly dependent to their water equivalent diameter (WED) that Radimetrics calculates from CT to obtain SSDE. High values of WED depend on patient size [23], then these data refer to obese patients, which seem to be most susceptible to COVID-19 complications.

SSDE is related to WED along the $\mathrm{z}$-axis and so it reflects the size variation that is present in our patient population, moreover the single patient size can change along the z-axis of a CT scan in the thorax region due to changes in body shape and attenuation at different areas, both these effects are not included in CTDIvol evaluation but can be explored looking at the SSDE frequency distribution. As expected, SSDE has an average value greater than CTDIvol and all the frequencies are shifted towards the right.

In a recent work by Agostini et al. [24], ultra low dose protocol with an effective dose lower than $1 \mathrm{mSv}$ is compared to high-resolution low dose protocol with COVID-19 positive patients in terms of diagnostic accuracy. In spite of the small sample size (8 patients), the authors conclude that the two protocols are comparable for patient diagnosis. In our institution radiologists have decided to use the high-resolution low dose protocols instead of ultra low dose protocols because the former guaranteed better delineation of the ground glass opacities typical of COVID-19 pneumonia [25]. Although it is quite clear that both protocols are suitable for solid nodules $>3 \mathrm{~mm}$ [26] there is no general consensus 


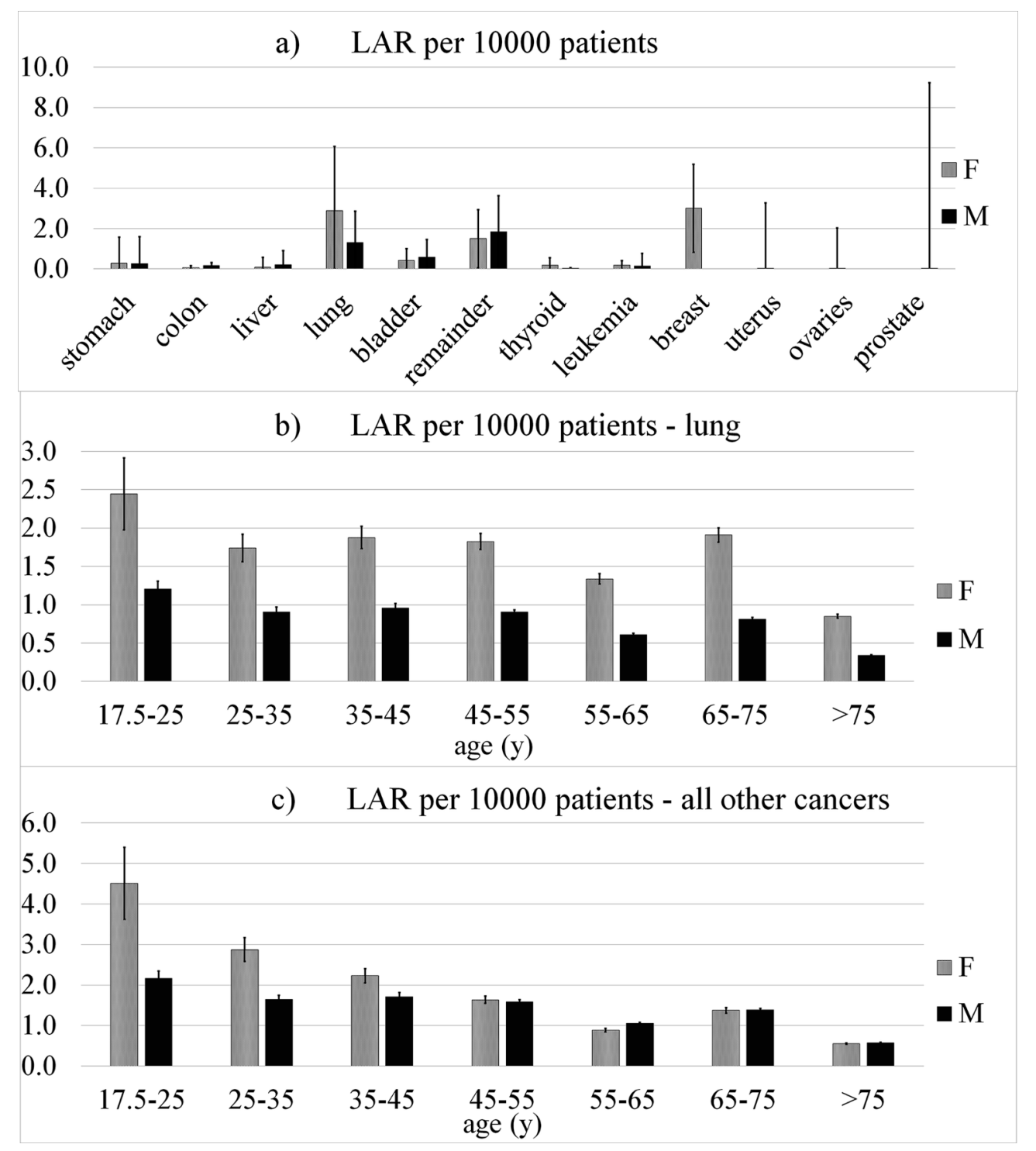

Fig. 4. Lifetime attributable risk per 10,000 patients subjected to CT examination for COVID-19 infection. Fig. 4.a show LAR of each cancer site for females and for males. Fig. 4.b reports LAR estimates of lung cancer for different age categories, while Fig. 4.c shows LAR estimates for all other cancer types. The 95\% confidence intervals are reported for all LAR estimates.

on the use of ultra low dose protocols for pulmonary emphysema, ground-glass opacity nodules or micro nodules in particular for patients with a BMI $>25 \mathrm{~kg} / \mathrm{m}^{2}$ [25] because high BMI influences the level of image noise on CT scans.

In order to evaluate the impact of radiation exposure connected to these diagnostic exams, the BEIR VII model was applied to calculate the risk of cancer induction. Using table 12-4 of the BEIR VII report it is possible to compare the cancer incidence due to radiation exposure of COVID-19 CT examinations (Fig. 4.a) to spontaneous cancer incidence. Cancer incidences due COVID-19 CT examinations were more than one order of magnitude greater than spontaneous cancer incidences for all cancer sites except to the farthest sites from the (thorax) scanning region, i.e. colon, uterus, ovaries and prostate. In particular, lung cancer incidence was up to 20-50 times greater. However, cancer incidences obtained in the present study by applying BEIR VII report were overestimated for many reasons that are discussed below.

The risk of developing radiation induced lung cancer was higher for women than for men by a factor that ranged between 1.9 and 2.5 depending on the age category (Fig. 4.b). This difference was probably due to a greater lung sensitivity to radiation for the women. In fact, the lung risk estimate in table 12D-1 of BEIR VII is 2.3 times higher for female than for male at all ages, while equivalent doses to the lungs are almost similar.

The risk of lung cancer seemed to decrease in both genders while the age of exposure increased. Age dependence was clearly evident only for first (17.5y-25y) and last ( $>75 y$ ) age categories with respect to the other categories. It is important to notice that only a few cases belong to the first age category: 24 for male and 13 for female.

As expected, lung cancer was the main disease which could be induced by diagnostic exposures for both genders (Fig. 4.a). Breast cancer risk was considerable for female also. For women, the risk of lung or breast cancer induction exceeded the risk of any other cancer by a factor of 6.9 or more. For men, the risk of lung exceeded the risk of any other cancer by a factor of 2.3 or more. The second site in order of greater LAR was the bladder in men. Results reported in Fig. 4.a are systematically overestimated, due to the fact that risk estimates used in this case are not age-specific and they refer to the generic U.S. population exposed to $0.1 \mathrm{~Gy}$. The mean age of COVID-19 patients analyzed in the present study was equal to 67 years, thus much higher than the mean age of the U.S. population described in the BEIR VII report. The relative 
importance of breast cancer risk respect to other type of cancer is overestimated in Fig. 4.a also, because the breast cancer risk has the strongest age dependence. For example, from table 12D-1, the risk estimate for breast decrease by a factor of 36 from age $20 \mathrm{y}$ to age $70 \mathrm{y}$, while the same factor for the lung risk estimate is only 2.4 .

The merit of Fig. 4.a is to provide a more reliable quantification of confidence intervals. The huge extent of confidence intervals is almost exclusively due to risk estimate uncertainty of the BEIR VII report (not to equivalent dose uncertainty) and attests that risk evaluation is only a very approximate estimation.

From Fig. 4.c, LARs of all other cancers rapidly decreased as the age of exposure increase, particularly for women. LAR estimates for females exceeded LAR estimates for males at younger ages (until the 35y-45y age category). Instead, the risk of induction of all other cancers was similar at older ages between men and women.

The assessment of cancer risk, according to the BEIR VII, is based on the linear no-threshold model and data collected from different populations living near nuclear facilities, exposed to high natural background radiation, exposed from Chernobyl and atomic bomb survivors [13]. The risk estimates of the BEIR VII report are thus an extrapolation at low doses using the linear no-threshold model for radiation risk, based on risk models generated from studies high level of radiation exposure. Several works have discussed the weakness of this approach, both for the use of linear no-threshold model and for the differences between populations $[27,28]$. All authors underlined that BEIR VII approach overestimates risk of cancer induction at lower doses that are typical of X-ray diagnostics. Moreover the application of the BEIR VII risk estimates is not recommended on a population affected by a pathology such as COVID-19. Even with this limitation, due to the sudden and considerable increase of CT examinations for COVId19 pandemic, it is interesting to provide an estimation, as rough as it may be, of the dosimetric impact of such diagnostic tool.

In spite of all these weaknesses, cancer risk estimation according to the BEIR VII allows a conservative approach that needs to be balanced with diagnostic benefits of medical imaging.

\section{Conclusion}

In this work we have demonstrated that despite the huge increment in thoracic CT examinations due to COVID-19 outbreak, the highresolution low dose protocol used in our hospital guaranteed to stay well beneath to national and international DRLs. Furthermore, we can conclude that although we have used a risk estimation model that overestimates the cancer induction, the results obtained in terms of LAR are low and the diagnostic benefits of chest CT examinations are well justified in these patients.

\section{Declaration of Competing Interest}

The authors declare that they have no known competing financial interests or personal relationships that could have appeared to influence the work reported in this paper.

\section{Appendix A. Supplementary data}

Supplementary data to this article can be found online at https://doi. org/10.1016/j.ejmp.2020.10.018.

\section{References}

[1] Huang C, Wang Y, Li X, et al. Clinical features of patients infected with 2019 novel coronavirus in Wuhan. China. Lancet 2020;395(10223):497-506. https://doi.org/ 10.1016/s0140-6736(20)30183-5.

[2] Novel Coronavirus (2019-nCoV) situation reports. 2020. https://www.who.int/ emergencies/diseases/novel-coronavirus-2019/situation-reports.

[3] | CDC. Interim Guidance: Healthcare Professionals 2019-nCoV 2020.
[4] Yang Y, Yang M, Shen C, et al. Evaluating the accuracy of different respiratory specimens in the laboratory diagnosis and monitoring the viral shedding of 2019nCoV infections. 2020. doi: 10.1101/2020.02.11.20021493.

[5] Ai T, Yang Z, Hou H, et al. Correlation of Chest CT and RT-PCR Testing in Coronavirus Disease, (COVID-19) in China: A Report of 1014 Cases. Radiology 2019;2020:200642. https://doi.org/10.1148/radiol.2020200642.

[6] Caruso D, Zerunian M, Polici M, Pucciarelli F, Polidori T, Rucci C, et al. Chest CT Features of COVID-19 in Rome. Italy Radiology 2020;3:201237. https://doi.org/ 10.1148/radiol.2020201237.

[7] Y. Fang H. Zhang J. Xie et al. Sensitivity of Chest CT for COVID-19: Comparison to RT-PCR Radiology 2020:200432. 10.1148/radiol.2020200432.

[8] J.P. Kanne B.P. Little J.H. Chung et al. Essentials for Radiologists on COVID-19: An Update- Radiology Scientific Expert Panel Radiology 2020:200527. 10.1148/ radiol.2020200527.

[9] Revel MP, Parkar AP, Prosch H, Silva M, Sverzellati N, Gleeson F, et al. European Society of Radiology (ESR) and the European Society of Thoracic Imaging (ESTI). COVID-19 patients and the radiology department - advice from the European Society of Radiology (ESR) and the European Society of Thoracic Imaging (ESTI). Eur Radiol. 2020. https://doi.org/10.1007/s00330-020-06865-y.

[10] Sverzellati N, Milanese G, Milone F, Balbi M, Ledda RE. Silva M Integrated Radiologic Algorithm for COVID-19 Pandemic. J Thorac Imaging. 2020 Apr 7. https://doi.org/10.1097/RTI.0000000000000516.

[11] Zhen Kang, Xu Li, Shuchang Zhou Recommendation of low-dose CT in the detection and management of COVID-2019 European Radiology 2020 https://doi. org/10.1007/s00330-020-06809-6.

[12] Berrington de González A, Mahesh M, Kim KP, Bhargavan M, Lewis R, Mettler F, et al. Projected cancer risks from computed tomographic scans performed in the United States in 2007. Arch Intern Med. 2009 Dec 14;169(22):2071-7. https://doi. org/10.1001/archinternmed.2009.440.

[13] Committee to Assess Health Risks from Exposure to Low Levels of Ionizing Radiation, National Research Council. Health Risks From Exposure to Low Levels of Ionizing Radiation: BEIR VII- Phase 2. National Academies Press; Washington, DC: 2005.

[14] International Commission on Radiological Protection, ICRP publication 103, Ann ICRP (2007).

[15] International Commission on Radiological Protection, ICRP publication 135. Ann ICRP (2017).

[16] Ghetti C, Ortenzia O, Palleri F, Sireus M. Definition of Local Diagnostic Reference Levels in a Radiology Department Using a Dose Tracking Software. Radiat Prot Dosimetry. 2017;175(1):38-45. https://doi.org/10.1093/rpd/ncw264.

[17] Palorini F, Origgi D, Granata C, Matranga D, Salerno S. Adult exposures from MDCT including multiphase studies: first Italian nationwide survey. Eur. Radiol. 2014;24:469-83.

[18] Smith-Bindman R, Moghadassi M, Wilson N, et al. Radiation doses in consecutive CT examinations from five university of California medical centers. Radiology 2015;277:134-41.

[19] Foley SJ. Mcentee MF and Rainford LA Establishment of CT diagnostic reference levels in Ireland. Br. J. Radiol. 2012;85:1390-7.

[20] Federal Office for Radiation Protection Notice of diagnostic reference levels for radiology and nuclear medicine examinations (Salzgitter, Germany: Federal Office for Radiation Protection) (2010).

[21] Santos J, Foley S, Paulo G, McEntee MF, Rainford L. The establishment of computed tomography diagnostic reference levels in Portugal. Radiat. Prot. Dosim. 2014;158:307-17.

[22] Padovani R, Compagnone G, D'Ercole Orlacchio A, Bernardi G, Rosi A, Campanella F. Livelli diagnostici di riferimento nazionali per la radiologia diagnostica e interventistica (in Italian). Rapporti Istisan 17/33. Istituto Superiore di Sanità. Roma 2017.

[23] Wang J, Christner JA, Duan X, Leng S, Yu L, McCollough CH. Attenuation-based estimation of patient size for the purpose of size specific dose estimation in CT. Part II. Implementation on abdomen and thorax phantoms using cross sectional CT images and scanned projection radiograph images. Med Phys. 2012 Nov;39(11): 6772-8. https://doi.org/10.1118/1.4757586.

[24] Agostini A, Floridi C, Borgheresi A, Badaloni M, Esposto Pirani P, Terilli F, et al. Proposal of a low-dose, long-pitch, dual-source chest CT protocol on thirdgeneration dual-source CT using a tin filter for spectral shaping at $100 \mathrm{kVp}$ for CoronaVirus Disease 2019 (COVID-19) patients: a feasibility study. Radiol Med. 2020 Apr;125(4):365-73. https://doi.org/10.1007/s11547-020-01179-x. Epub 2020 Apr 1.

[25] Kim Y, Kim YK, Lee BE, Lee SJ, Ryu YJ, Lee JH. Chang JH Ultra-Low-Dose CT of the Thorax Using Iterative Reconstruction: Evaluation of Image Quality and Radiation Dose Reduction. AJR Am J Roentgenol. 2015 Jun;204(6):1197-202. https://doi. org/10.2214/AJR.14.13629.

[26] Paks M, Leong P, Einsiedel P, Irving LB, Steinfort DP, Pascoe DM. Ultralow dose CT for follow-up of solid pulmonary nodules: A pilot single-center study using BlandAltman analysis. Medicine (Baltimore). 2018 Aug;97(34):e12019. https://doi.org/ 10.1097/MD.0000000000012019.

[27] Hendee WR, O'Connor MK. Radiation risks of medical imaging: separating fact from fantasy. Radiology 2012;264:312-21. https://doi.org/10.1148/ radiol.12112678.

[28] O'Connor MK. Risk of low-dose radiation and the BEIR VII report: A critical review of what it does and doesn't say. Phys Med. 2017 Nov;43:153-8. https://doi.org/ 10.1016/j.ejmp.2017.07.016. Epub 2017 Aug 18. 